\title{
Opportunities and Challenges in the Application of Advanced Control to Power Electronics and Drives
}

\author{
Graham C. Goodwin*, David Q. Mayne ${ }^{\dagger}$, Tina Chen ${ }^{\ddagger}$, Colin Coates*, Galina Mirzaeva* and Daniel E. Quevedo* \\ *School of Electrical Engineering and Computer Science, The University of Newcastle, Callaghan, NSW 2308, Australia. \\ Email: graham.goodwin@newcastle.edu.au \\ ${ }^{\dagger}$ Department of Electrical and Electronic Engineering, Imperial College, London, SW7 2BT, England, UK. \\ Email: d.mayne@imperial.ac.uk

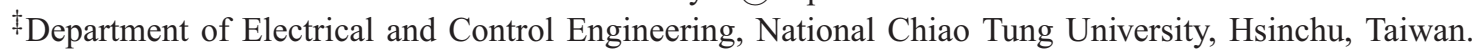 \\ Email: bettery33@gmail.com
}

\begin{abstract}
Control technology underpins the operation of many, and arguably all, modern high technology systems. Such systems include transportation (aircraft, high speed trains, marine vessels, automobiles), telecommunication systems, electricity networks, mining, minerals processing and agriculture. A particular area where control is playing an increasingly important role is industrial electronics. In this paper we will adopt a control engineering perspective to reflect on the opportunities and challenges that exist in the application of advanced control to these systems.
\end{abstract}

\section{INTRODUCTION}

Advanced control is a mature subject. It has gone through various phases of development (45) including Classical Control, $L_{2}$ optimal control, robust control and, most recently, networked control and quantum control. Often it has been new application areas that have inspired control theory developments. For example, the ideas of network control are responding to the challenges arising from implementing control over a telecommunication network. This problem leads to new questions regarding the impact of limited channel capacity, random delays and lost packets, (19), (20). Also, research in quantum control is inspired by the potential of quantum computing.

A technology that underpins the operation of many modern systems is that of switching electronics. This technology lies at the core of many new systems including smart electricity grids, wind and solar power, high speed trains and electric and hybrid vehicles. These systems give rise to a unique set of control engineering opportunities and challenges. Certainly many of these challenges have already been addressed or are the subject of current research, (1), (2), (3). In this context, the aim of the current paper is to take a longer term view and ask which issues control theoreticians might look at to better support current and future advances in industrial electronics.

An overview of the remainder of the paper is as follows: In section II, we briefly review control problems in power electronics and drives. In section III, we give a brief overview of key concepts in control theory. In section IV, we illustrate the ideas of section III by reference to the control of a Synchronous Reluctance Machine. In section V, we provide a brief overview of Model Predictive Control. In section VI we discuss issues involved in providing a "certificate of stability" and we introduce the idea of "Fusion Control". In section VII, we discuss challenges and opportunities. Finally, in section VIII, we draw conclusions.

\section{Brief Overview of Control Problems in Power ELECTRONICS AND DRIVES}

\section{A. General Comments}

In the area of power electronics and drives, a wide range of circuit topologies are used in various applications. There are many different types of switch mode converter and inverter circuits that find use in power supply, motor drives and power conditioning applications.

Although the range of circuit architectures is diverse, it is also true that the associated control problems share many common elements. The controllers generally seek to regulate a voltage or current with minimum steady state error while maintaining a fast transient response. In each instance, the controller output is limited to a finite set of values determined by the circuit's switching states. The controller must be robust, remaining insensitive to variation in plant parameters. For example, the inductance and resistance values in motor drives can vary significantly due to magnetic saturation and thermal effects. Also, one typically wants to minimize excessive switching to enhance efficiency. Finally, allowance must be made for the noise content in feedback signals as power electronic circuits are inherently noisy.

\section{B. Motivational Example}

The torque control of a synchronous reluctance motor (SynRM) is used here as a representative problem to demonstrate some of the key control issues in power electronics and drive circuits. The example is also of interest in its own right. Although, the induction motor (IM) is the most popular machine for industrial use, the modern SynRM is considered to be a viable alternative. While providing a torque comparable to that provided by an IM, the losses in a SynRM are about $50 \%$ of those in an IM. Thus, in the "energy efficient" future, it is expected that the SynRM will receive increasing attention.

The SynRM is distinguished by its rotor structure where axial laminations, or other techniques, are used to produce 
both a low reluctance and high reluctance magnetic flux path, (43). By convention, the low reluctance axis is called the direct (D) axis and the high reluctance axis is called the quadrature (Q) axis. The stator of a SynRM is typically a three-phase distributed winding as is common to other AC machines. The SynRM develops torque as it seeks to align its low reluctance axis to the stator field.

The control of a SynRM is usually addressed in a rotating DQ reference frame which is fixed to the rotor. This offers the advantage of transforming the $\mathrm{AC}$ stator current variables to $\mathrm{DC}$ values. Additionally, the transformation removes the position dependence and mutual inductance terms otherwise obtained when operating in stator variables. The voltage and torque equations for a three-phase SynRM in the rotor DQ reference frame are well known (43):

$$
\begin{aligned}
v_{d} & =R_{s} i_{d}+L_{d} \frac{d i_{d}}{d t}-\omega L_{q} i_{q} \\
v_{q} & =R_{s} i_{q}+L_{q} \frac{d i_{q}}{d t}+\omega L_{d} i_{d} \\
T & =\frac{3 p}{2}\left(L_{d}-L_{q}\right) i_{d} i_{q}
\end{aligned}
$$

Figure 1 shows the typical control structure for a SynRM drive (48). An outer speed control loop generates a torque reference which translates into the quadrature current reference using (3). Speed in the outer loop can be measured by a sensor (as shown) or estimated using the natural SynRM rotor saliency. The combined direct and quadrature currents are controlled by an inner current control loop that determines the voltage to be applied to the motor in the DQ reference frame. The direct axis current reference is set to its rated value. This ensures the machine is fully fluxed and maximum torque is obtained. In a three-phase drive only eight (seven independent) voltage vectors can be applied to the motor via the inverter. The switching selector chooses the most appropriate voltage vector or sequence of voltage vectors to apply to the machine at each control cycle.

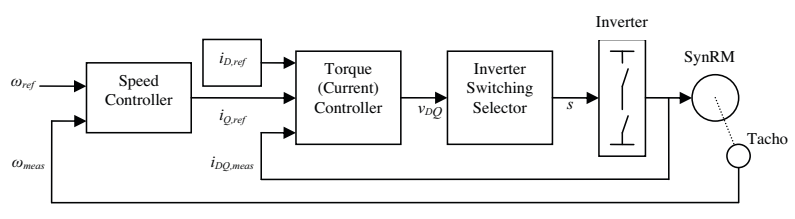

Fig. 1. Synchronous Reluctance Motor Drive

The most common approaches to implementing the current control loop and inverter switching selection blocks are as follows (4):

a. Hysteresis Control: The direct and quadrature current references can be transformed to stator phase current values. These reference values are then compared to the actual stator phase currents. The individual inverter legs are switched to maintain the associated phase current within a band around the reference value (44). While this type of controller is simple to implement, it requires higher switching frequencies leading to increased inverter switching losses.

b. Linear Control with PWM: The error between the reference and measured values of direct and quadrature currents are fed to respective controllers (typically PI) that generate reference values for the direct and quadrature axis voltages. A pulse width modulator is then used to generate the switching signals for the inverter (46).

c. Predictive Control Methods: Given the current state of the machine, the motor voltage equations can be used to determine the ideal direct and quadrature voltages to move the current vector to its desired value in one step. In this instance, the switching selector block chooses the inverter switching configuration (or a combination of switching configurations) that is closest to approximating the ideal voltage vector. This switching configuration is applied over the next control cycle, (44), (4).

d. Other Control Techniques e.g., Fuzzy logic and sliding mode.

We will examine a particular multi-degree-of-freedom control architecture in section IV for a 9 phase SynRM.

\section{A BRIEF OVERVIEW OF CONTROL THEORY}

\section{A. Inversion}

At a high level of abstraction, we can view the problem of control as one of inversion (45). One has a system (usually called the "plant") which has outputs (process variables). The plant is acted upon by inputs (manipulated variables). The core problem is to choose the inputs so that the outputs achieve certain objectives. If one has a model linking inputs to outputs, then one sees that the above problem can, in principle, be solved by inverting the model so as to evaluate the necessary inputs which bring the outputs to their desired values. Thus, conceptually, if the model takes the form:

$$
y=G(u)
$$

where $y, u$ denote the output and input respectively, and we want $y$ to be $y^{*}$, then the required input is simply given by

$$
u^{*}=G^{-1}\left(y^{*}\right)
$$

Of course, there are a number of practical issues which render this solution too naive in most real situations. Some of the additional features of real problems include (45):

(i) Disturbances: The output response $y$ is influenced by (load) disturbances in addition to the input, $u$.

(ii) Measurement errors: It is helpful, at least intuitively, to know if $y$ has reached the target value $y^{*}$. However, the measurements of $y$ are typically corrupted by noise and other measurement imperfections.

(iii) Input Constraints: It is usual that we simply cannot apply any desired input to the plant. For example, in AC drives, the input will typically be restricted by the finite set of available switch positions. Thus one must "make the best use of" the available input options. 
(iv) Dynamics: The model (4) implies that the effect of changing the input is instantaneous on the output. However, all real systems are subject to energy storage and delays i.e., the voltage in capacitors and the current in conductors cannot be changed instantly.

(v) Model errors: Typically the model $G(\cdot)$ is, at best, only approximately known. Hence one needs to find a way of obtaining an inverse which is insensitive to model errors.

(vi) Time variations: Typically the model $G(\cdot)$ will not be fixed, e.g., Resister and inductor values can change as the temperature changes.

(vii) Robustness: This is a term used to describe control systems that operate satisfactorily in the light of the kind of problems mentioned under (v) and (vi) above.

In the sequel we will use a slightly more general way of modelling the system. We will use a transfer function description based on z-transforms.

$$
\begin{aligned}
y(z) & =G(z) u(z)+d_{0}(z) \\
y_{m}(z) & =y(z)+n(z)
\end{aligned}
$$

where $n, d_{0}$ represent (the z-transform of) measurement noise and process disturbances (lumped at the output).

\section{B. High Gain Feedback}

It turns out that a remarkably robust way of achieving "inversion" is via feedback. Thus, if we have a desired value, $y^{*}$, for $y$, then we might envisage generating the input via a feedback law of the form:

$$
u(z)=-C(z) y_{m}(z)+M^{\prime}(z) y^{*}(z)
$$

To simplify developments we will temporarily restrict attention to single-input single-output systems. (The results apply more generally.) We also choose $M^{\prime}(z)=C(z)$. (Other choices will be explored in section III-F).

Solving (6) to (8) simultaneously gives the following "closed loop" relationship

$$
\begin{aligned}
y(z)= & \frac{G(z) C(z)}{1+G(z) C(z)} y^{*}(z) \\
& -\frac{G(z) C(z)}{1+G(z) C(z)} n(z) \\
& +\frac{1}{1+G(z) C(z)} d_{0}(z)
\end{aligned}
$$

Equation (9) motivates us to introduce two key closed loop transfer functions $T(z)$ and $S(z)$. These are called the complementary sensitivity and sensitivity respectively:

$$
\begin{aligned}
T(z) & =\frac{G(z) C(z)}{1+G(z) C(z)} \\
S(z) & =\frac{1}{1+G(z) C(z)}
\end{aligned}
$$

We can then write

$$
y(z)=T(z) y^{*}(z)-T(z) n(z)+S(z) d_{0}(z)
$$

We now see how feedback is a way of achieving inversion. All we need do is make $C(z)$ "very large" to achieve

$$
T(z)=1
$$

In this case, the "closed loop" transfer function from $y^{*}$ to $y$ becomes 1 and $u(z)=G(z)^{-1}\left[y^{*}(z)-d_{0}(z)\right]$ as required.

\section{Design Trade-offs}

Alas, the above (high gain) solution to the control problem isn't as easy as it sounds. For example, (9) shows that putting $T(z)=1$, causes the measurement noise to be transferred to the output $y(z)$. Another slightly more subtle issue is that we see from $(10),(11)$ that

$$
T(z)+S(z)=1
$$

Hence, if we want low sensitivity to measurement noise $(T(z) \rightarrow 0)$, then necessarily we have $100 \%$ sensitivity to (output) disturbances $(S(z) \rightarrow 1)$ and vice versa.

Actually, there is another reason why we may not want to have infinite (or a least very high) gain in the controller. The reason is that we usually only have an approximate model. To illustrate, let us describe model errors in the frequency domain by saying that the true plant transfer function, $G(z)$ is related to the given model, $G_{0}(z)$, by a relationship of the form:

$$
G\left(e^{j \omega \triangle_{s}}\right)=G_{0}\left(e^{j \omega \triangle_{s}}\right)\left[1+G_{\triangle}\left(e^{j \omega \triangle_{s}}\right)\right]
$$

where $\triangle_{s}$ is the sampling period.

We say that $G_{\triangle}\left(e^{j \omega \triangle_{s}}\right)$ is the multiplicative error at frequency $\omega$.

One can then easily derive the following relationship between the true sensitivity function $S\left(e^{j \omega \triangle_{s}}\right)$ and the nominal sensitivity function $S_{0}\left(e^{j \omega \triangle_{s}}\right)$ :

$$
\begin{aligned}
S_{0}\left(e^{j \omega \triangle_{s}}\right) & =\frac{1}{1+G_{0}\left(e^{j \omega \triangle_{s}}\right) C\left(e^{j \omega \triangle_{s}}\right)} \\
S\left(e^{j \omega \triangle_{s}}\right) & =\frac{S_{0}\left(e^{j \omega \triangle_{s}}\right)}{1+T_{0}\left(e^{j \omega \triangle_{s}}\right) G_{\triangle}\left(e^{j \omega \triangle_{s}}\right)}
\end{aligned}
$$

Say one designs $S_{0}\left(e^{j \omega \triangle_{s}}\right)$ to be small (over some frequency range), then $T_{0}\left(e^{j \omega \triangle_{s}}\right)$ will be near unity over the same frequency range. Thus we see that there is a potential danger arising from $G_{\triangle}\left(e^{j \omega \triangle_{s}}\right)$. Indeed, a sufficient condition for closed loop stability is that $\left|T_{0}\left(e^{j \omega \triangle_{s}}\right)\right|\left|G_{\triangle}\left(e^{j \omega \triangle_{s}}\right)\right|$ should be less than 1 at all frequencies. This is a simple consequence of (17).

Often, in practical models, $\left|G_{\triangle}\left(e^{j \omega \triangle_{s}}\right)\right|$ will be large at high frequency. Thus robustness to model errors typically places an upper band on the frequencies over which $\left|T_{0}\left(e^{j \omega \triangle_{s}}\right)\right|$ can be kept near 1 . We call this range of frequencies the "closed loop bandwidth".

There are other reasons why one cannot make $S_{0}\left(e^{j \omega \triangle_{s}}\right)$ small at all frequencies. These constraints arise from the need to make $S_{0}\left(e^{j \omega \triangle_{s}}\right)$ stable i.e., to keep $S_{0}(z)$ an analytic function. In a key result, Bode showed that for all analytic functions, we have (45)

$$
\int_{-\frac{\pi}{\Delta_{s}}}^{\frac{\pi}{\triangle_{s}}} \log \left|S_{0}\left(e^{j \omega \triangle_{s}}\right)\right| d \omega \geqslant 0
$$


Of course, $\log |a| \geqslant 0$ if $a \geqslant 1$. Hence, (18) tells us that there is a remarkable "water bed" effect i.e., if we have a range of frequencies where $\left|S_{0}\left(e^{j \omega \triangle_{s}}\right)\right|<1$, then there must be another range of frequencies where $\left|S_{0}\left(e^{j \omega \triangle_{s}}\right)\right|>1$.

\section{State Estimate Feedback}

In modern control it is common to replace the transfer function model of (6) by a state variable model of the form:

$$
\begin{aligned}
x^{+} & =A x+B u \\
y & =C x
\end{aligned}
$$

where $x^{+}$is the next value of $x$.

If the state were directly measured, then one could design state variable feedback of the form:

$$
u=-K x+M(z) y^{*}
$$

Subject to mild assumptions (e.g. controllability) then $K$ can be chosen to give desired closed loop properties. (e.g., specified closed loop poles.)

However $x$ is not usually directly measured. In this case one can obtain an estimate of $x$ by using an observer:

$$
\hat{x}^{+}=A \hat{x}+B u+J(y-C \hat{x})
$$

and implement the control law as

$$
u=-K \hat{x}+M(z) y^{*}
$$

We can actually reinterpret the controller given in (22), (23) in the Classical Transfer Function form as in sections III-B and III-C by taking z-transforms in (22). This leads to

$$
u=-T_{1}(z) u-T_{2}(z) y+M(z) y^{*}
$$

where

$$
\begin{aligned}
& T_{1}(z)=K(z I-A+J C)^{-1} B=: \frac{R(z)}{E(z)} \\
& T_{2}(z)=K(z I-A+J C)^{-1} J=: \frac{P(z)}{E(z)}
\end{aligned}
$$

Rearranging (24) we obtain:

$$
u=-C(z) y+M^{\prime}(z) y^{*}
$$

where $C(z)=P(z) /[R(z)+E(z)]=: P(z) / L(z) ; M^{\prime}(z):=$ $E(z) M(z) / L(z)$.

Thus we have given a modern interpretation (in the form of state estimate feedback) to the classical control law given in (8).

\section{E. Internal Models}

An important practical issue when designing feedback control laws is to ensure that certain disturbances are exactly cancelled at the output. We can achieve this goal by including a model for the disturbances in the description (19).

To explain this idea in more detail, say that we have an input disturbance $d_{i}$. We can model $d_{i}$ as the output of a state space model as shown in (28). The true input to the plant is $u+d_{i}$.

$$
\begin{aligned}
x_{d}^{+} & =A_{d} x_{d} \\
d_{i} & =C_{d} x
\end{aligned}
$$

Common choices for $A_{d}$ would be a scalar matrix consisting of unity (to describe a constant disturbance) or 2 dimensional matrix having eigenvalues on the unit disc (to describe a sinusoidal disturbance of a given frequency).

The system matrices for the observer and associated feedback control law then take the form:

$$
\begin{aligned}
A & =\left[\begin{array}{cc}
A_{0} & B_{0} C_{d} \\
0 & A_{d}
\end{array}\right] ; B=\left[\begin{array}{c}
B_{0} \\
0
\end{array}\right] \\
J & =\left(\begin{array}{c}
J_{0} \\
J_{d}
\end{array}\right) \quad ; C=\left[\begin{array}{ll}
C_{0} & 0
\end{array}\right] \\
K & =\left[\begin{array}{ll}
K_{0} & C_{d}
\end{array}\right]
\end{aligned}
$$

Notice that this control law cancels the estimated disturbance at the input.

It can readily be shown that, in this case, $L(z)=R(z)+$ $E(z)$ is given by

$$
\begin{aligned}
L(z) & =\operatorname{det}[z I-A+J C+B K] \\
& =\operatorname{det}\left[\begin{array}{cc}
z I-A_{0}+J_{0} C_{0}+B_{0} K_{0} & -B_{0} C_{d}+B_{0} C_{d} \\
J_{d} C_{0} & z I-A_{d}
\end{array}\right] \\
& =\operatorname{det}\left[\begin{array}{cc}
z I-A_{0}+J_{0} C_{0}+B_{0} K_{0} & 0 \\
J_{d} C_{0} & z I-A_{d}
\end{array}\right] \\
& =\operatorname{det}\left(z I-A_{d}\right) \operatorname{det}\left(z I-A_{0}+J_{0} C_{0}+B_{0} K_{0}\right)
\end{aligned}
$$

Hence we conclude that $L(z)$ will be zero at the zeros of the disturbance model.

The significance of this observation will be made clear in the next section.

\section{F. Multi-degree of Freedom Control Laws}

The operation of a feedback control loop typically needs to deal with multiple (and often conflicting) design objectives. For example:

(1) We may want to minimize the impact of (unmeasured) load disturbances on the output. (This is governed by $S(z)=1 /[1+G(z) C(z)]$.

(2) We may want to minimize the impact of measurement noise on the output. (This is governed by $T(z)=$ $G(z) C(z) /[1+G(z) C(z)]$.

(3) We may want to track a given reference signal $y^{*}$. (Also, $y^{*}$ will typically be time varying.)

(4) We may want to minimize the impact of implementation errors (e.g. quantization) in $u(t)$ on the output.

1) Disturbances and Noise: The situation regarding points (1) and (2) is fundamentally constrained by the fact that

$$
S(z)+T(z)=1
$$

The linking of goals (1) and (2) is inescapable since the relevant part of the control law only uses the measurement $y$; i.e., it has only one-degree-of-freedom namely $C(z)$. 
The situation regarding points (2) and (3) is different because we have extra measurements that we can exploit.

2) Reference Tracking: We have already added on extra degree of freedom to the control law in (24) by including a transfer function $M(z)$. A typical choice for $M(z)$ is

$$
M(z)=\frac{P(z)}{E(z)}+\frac{L(z)}{E(z)} F(z)
$$

or equivalently $M^{\prime}(z)=C(z)+F(z)$.

The resultant transfer function from $y^{*}$ to $y$ now becomes

$$
T_{y^{*} y}(z)=1-\frac{L(z)[A(z)-F(z) B(z)]}{A(z) L(z)+B(z) P(z)}
$$

We also recall the transfer function from $d_{0}$ to $y$ which is

$$
S_{d_{0} y}(z)=\frac{A(z) L(z)}{A(z) L(z)+B(z) P(z)}
$$

Examination of (36), (37) reinforces the importance of the result derived earlier in (33), namely provided (28), (29) include a model for the disturbance and the reference, then perfect tracking is obtained irrespective of model errors since $L\left(z_{0}\right)=0$ where $z_{0}$ is a zero of the disturbance (and reference) model.

We also, see the advantages of having $F(z)$ in (36), namely we can reduce the tracking error at every frequency where $F(z)$ can be chosen as an approximate inverse to the plant at these frequencies, i.e.,

$$
F(z) \simeq\left[\frac{B(z)}{A(z)}\right]^{-1} ; \quad z=e^{j \omega \triangle_{s}}
$$

3) Input Imperfections: We next turn to point 4. This issue is of great importance in power electronics since the switched nature of the input restricts the allowed input to a finite set. We can describe this implementation "defect" via

$$
u(t)=Q\left[u_{0}(t)\right]
$$

where $u(t) \in \mathbb{U}$ (the allowable set), $u_{0}(t)$ is the desired input coming from the controller and $Q$ is a nonlinear mapping (a generalized "quantizer" (18)).

For design purposes, it is often helpful to think of the difference between $u_{0}(t)$ and $Q\left(u_{0}(t)\right)$ as a noise source, $q(t)$, where

$$
q(t)=Q\left(u_{0}(t)\right)-u_{0}(t)
$$

or

$$
u(t)=u_{0}(t)+q(t)
$$

We could rely upon the feedback controller, $C(z)$, to reduce the impact of $q$ on the output. However, this may compromise the design relative to the impact of $d_{0}$ and $n$. Hence, we introduce an extra degree of freedom by using a feedback quantizer as shown in figure 2 .

If we model the quantizer as a noise source as in (41), then with $H(z)=0$, we see that the local transfer function from $q(t)$ to $u(t)$ is 1 .

On the other hand, if we introduce the additional transfer function $H(z)$ then the transfer function from $q(t)$ to $u(t)$

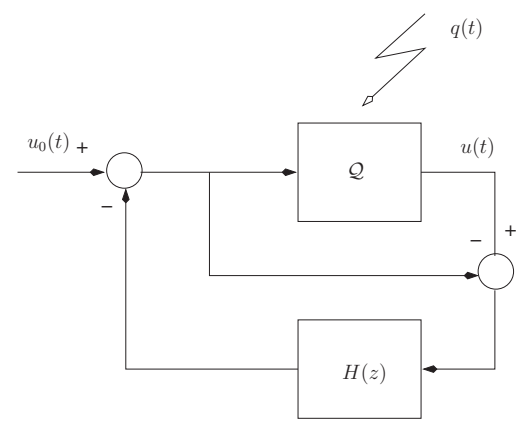

Fig. 2. Feedback Quantizer

becomes $1-H(z)$. Hence, we can choose $H(z)$ to "shape" the frequency content of the errors due to the quantizer. Most importantly, this can be achieved without compromising or affecting any of the other design objectives.

To illustrate, say we implement the controller digitally and we want to eliminate errors due to the quantizer at $\omega_{0}$.

Then we can choose

$$
H(z)=\frac{\left[2(1-\alpha) \cos \omega_{0} \triangle_{s}\right] z+\left(\alpha^{2}-1\right)}{z^{2}-\left(2 \alpha \cos \omega_{0} \triangle_{s}\right) z+\alpha^{2}}
$$

This leads to

$$
1-H(z)=\frac{z^{2}-\left(2 \cos \omega_{0} \triangle_{s}\right) z+1}{z^{2}-\left(2 \alpha \cos \omega_{0} \triangle_{s}\right) z+\alpha^{2}}
$$

This transfer function is zero at frequency $\omega=\omega_{0}$.

The effectiveness of this strategy in eliminating unwanted components arising from a quantizer is illustrated by the following example. We simulate a feedback control loop for a first order plant with integral action in the controller. We first use a simple (nearest neighbour) quantizer. Figure 3 shows the spectrum of the input signal in this case. It can be seen that there is a significant spectral peak near $45 \mathrm{~Hz}$. This might have undesirable practical consequences, e.g., it may excite a resonance in a motor. When we introduce an extra degree of freedom, via the filter $H(z)$ (see (42)) in the quantizer, then as shown in figure 4 , the unwanted spectral line disappears. Of course, the Bode integral (18) also holds for $S^{\prime}(z)=1-H(z)$. This implies that there must be a trade-off i.e., reduction of "quantization noise" at one frequency must be accompanied by an increase in "quantization noise" at other frequencies. This effect is also evident in figures 3 and 4. This idea can be used to eliminate (or at least reduce) any undesirable frequency component on the input (subject to satisfaction of the Bode integral trade-off).

\section{G. Constrained Observer Feedback (COF)}

A final problem that we wish to discuss is that of input saturation. Specifically, a high gain linear feedback law is almost certain to call for larger inputs (e.g. voltages) than are available. this problem is central to many practical control problems.

A key approach to dealing with these problems is to make sure that the various controllers are "informed" that 


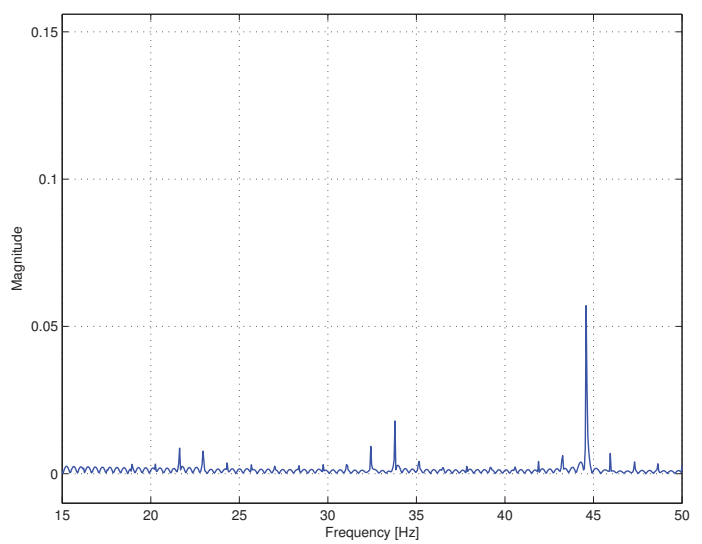

Fig. 3. Input Spectrum using Nearest Neighbour Quantizer

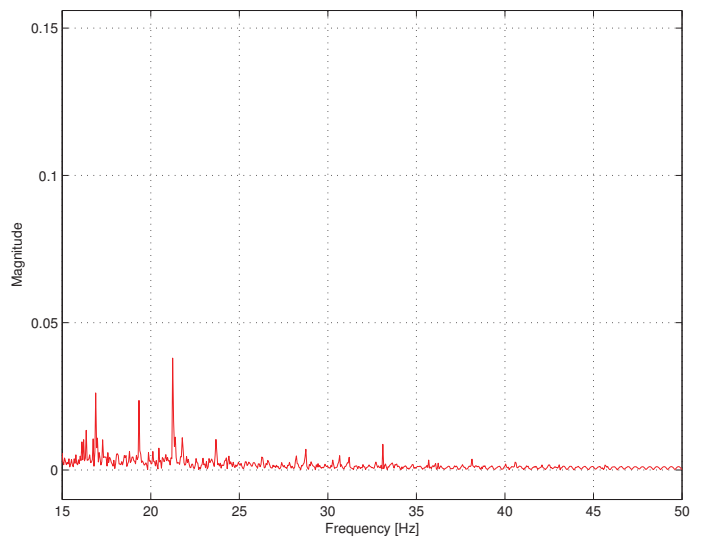

Fig. 4. Input Spectrum using Feedback Quantizer

the (hypothetical) input requested by the linear control law was not actually used. This concept turns out to be a (mild) generalization of well known ideas used in integral control to prevent integrator wind up. These techniques are usually called Anti-Wind-Up (AWU) Control (45).

We discuss this issue below for the feedback controller $C(z)=P(z) / L(z)$ and the feedforward controller, $F(z)$.

1) Feedback Controller: In this case, the solution is rather simple. All we need to do is to ensure that the observer part of the feedback controller "knows" about the true input rather than the hypothetical input generated by the linear controller.

Hence the appropriate circuit is as shown in figure 5:

2) Feedforward Controller: The situation with feedforward control is a little more subtle. To deal with this, we factor $F(z)^{-1}$ as follows:

$$
F(z)^{-1}=f_{0}+\bar{F}(z)
$$

where $\bar{F}(z)$ is strictly proper.

We then implement the feedforward controller as shown figure 6:

We see that, in the absence of quantization, or saturation,

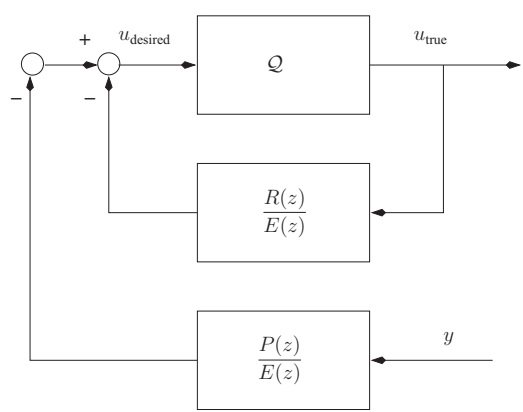

Fig. 5. COF Implementation of Feedback Controller

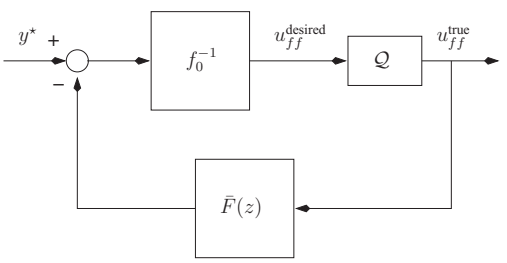

Fig. 6. COF Implementation of Feedforward controller

that

$$
\begin{aligned}
u_{f f}^{\text {true }} & =\frac{f_{0}^{-1}}{1+f_{0}^{-1} \bar{F}(z)} y^{*} \\
& =F(z) y^{*} \text { as required }
\end{aligned}
$$

However, in the presence of quantization, the circuit ensures that the states of the feedforward filter which appear in $\bar{F}(z)$, "know" that $u_{f f}^{\text {true }}$ was actually used rather than $u_{f f}^{\text {desired }}$.

\section{H. Composite Design}

Finally, we can put all of the elements together. One final point to be addressed is that $u$ comprises two signal i.e., $u_{f f}$ and $u_{f b}$.

Hence we define

$$
\lambda=\left|\frac{Q\left[u_{f f}^{\text {desired }}+u_{f b}^{\text {desired }}\right]}{\left[u_{f f}^{\text {desired }}+u_{f b}^{\text {desired }}\right]}\right|
$$

Then we calculated

$$
\begin{aligned}
& u_{f f}^{\text {actual }}=\lambda u_{f f}^{\text {desired }} \\
& u_{f b}^{\text {actual }}=\lambda u_{f b}^{\text {desired }}
\end{aligned}
$$

We can then draw the complete design as in figure 7.

\section{Illustration Via Control of Synchronous RELUCTANCE MOTOR}

To illustrate some of the ideas described above we will present results for the (simulated) control of a nine phase voltage source inverter driving a SynRM - see figure 8 and section II. We will focus on torque control with constant fluxing of the machine.

We will investigate the impact of the design and implementation (in COF form) of the feedback and feedforward loop. We use a sample period of $\triangle_{s}=0.2 \mathrm{msec}$. 


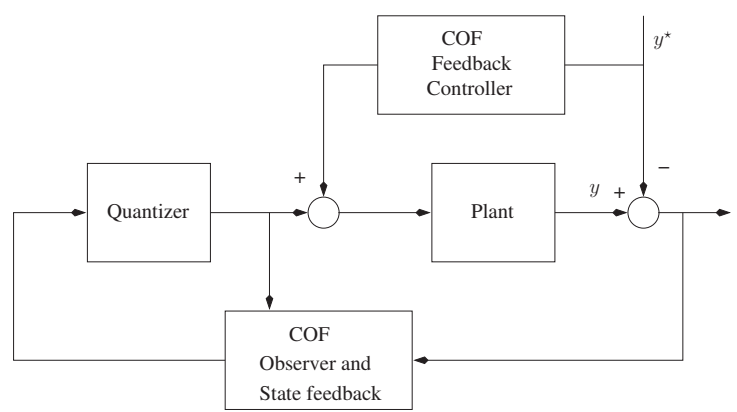

Fig. 7. COF Implementation of Complete Control Law

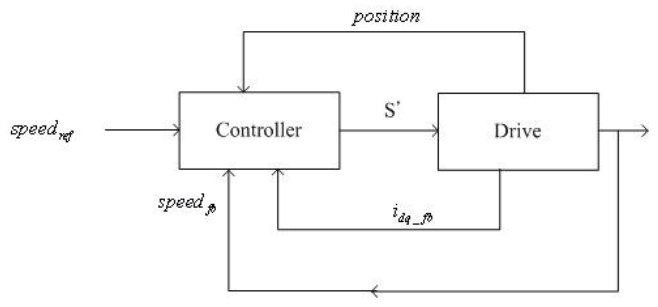

Fig. 8. SynRM Control

\section{A. The Feedback Controller}

For simplicity, we examine the locked rotor scenario. Comments on the non-locked case will be given in section VI-D. We assume a constant direct axis reference current of 2(A) and we measure all currents in the presence of measurement noise. Hence, in view of the comments made under heading (1) and (2) in section III-F, we will need to limit the feedback bandwidth so as to obtain an appropriate trade-off between disturbance rejection and noise immunity.

We utilize a simple PI controller where we cancel the plant pole by a zero in the controller and introduce integral action. This leads to

$$
C(z)=\frac{K_{p}(z-1)+K_{I} \triangle_{s} z}{(z-1)}
$$

where $K_{I}=(1+\gamma)(1-\alpha) /\left[\triangle_{s} \beta\right] ; K_{p}=\alpha(1-\gamma) / \beta$, where $\alpha$ is the open loop (discrete time) pole, $\gamma$ is the desired (discrete time) closed loop pole.

We implement the controller in $\mathrm{COF}$ form as shown in figure 9 where $C(z)^{-1}=c_{0}+\bar{C}(z), c_{0}^{-1}=\left(K_{p}+K_{I} \Delta_{s}\right)$, and where the quantizer is placed immediately after $c_{0}^{-1}$.

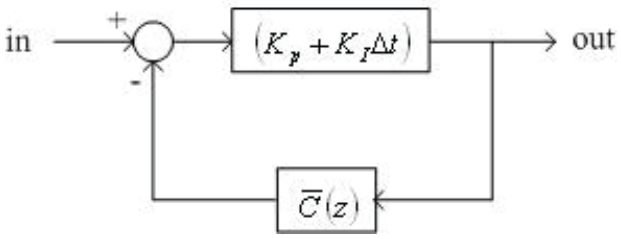

Fig. 9. COF Implementation of Feedback Controller

The quantizer is a mechanism which ensures that the voltage corresponds to one of the allowed switching states.

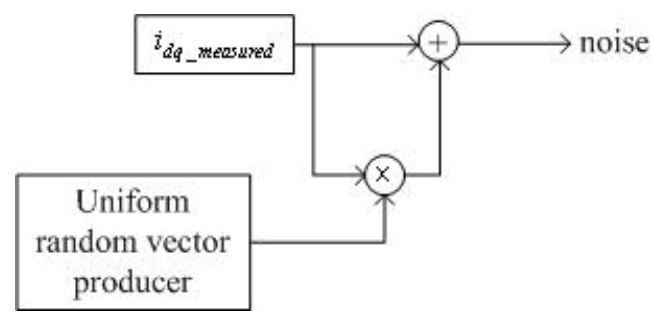

Fig. 10. Measurement Noise Generation

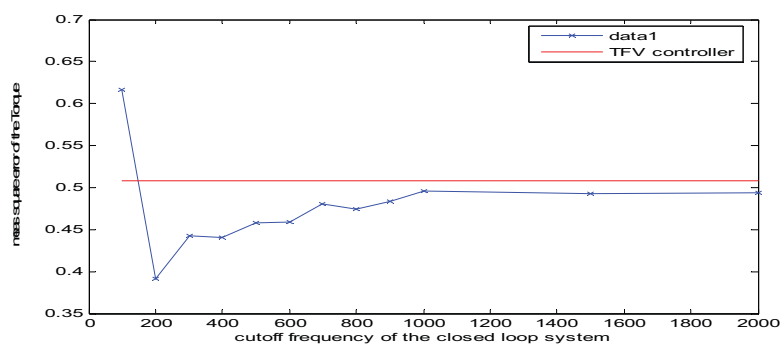

Fig. 11. Mean Square Error as a Function of Closed Loop Bandwidth

We simulate the measurement noise as uniformly distributed white noise in the interval $[-0.1,0.1]$. We then use this to give about $10 \%$ measurement noise as shown in figure 10 .

Figure 11 shows the mean square torque error as a function of closed loop bandwidth. We see that the optimal closed loop bandwidth is approximately $200 \mathrm{~Hz}$.

Next we vary the noise amplitude and plot the optimum bandwidth as a function of noise amplitude. The results are shown in figure 12. As expected, the optimum bandwidth decreases as we increase the measurement noise level.

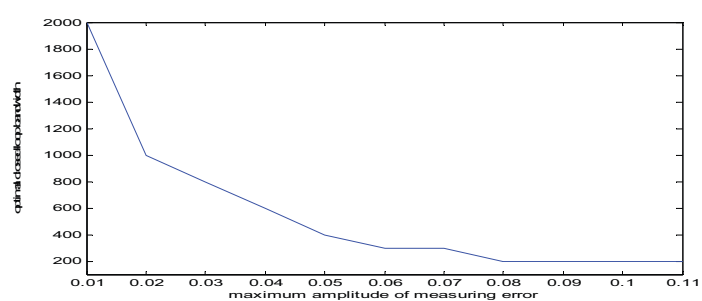

Fig. 12. Optimum Bandwidth as a Function of Measurement Noise Amplitude

Finally, we test the use of COF feedforward. We design the (unconstrained) response time of the feedforward to be $\frac{1}{5}^{\text {th }}$ that of the feedback loop. We use a set point change of $10 \%$ downwards after reaching steady state. (We choose a small reference change so that the results are not dominated by input slewing resulting from saturation effects.)

Figure 13 compares the measured response time for 3 controllers as follows:

(i) TFV - this is the predictive control method described in part (b) of Section II. 


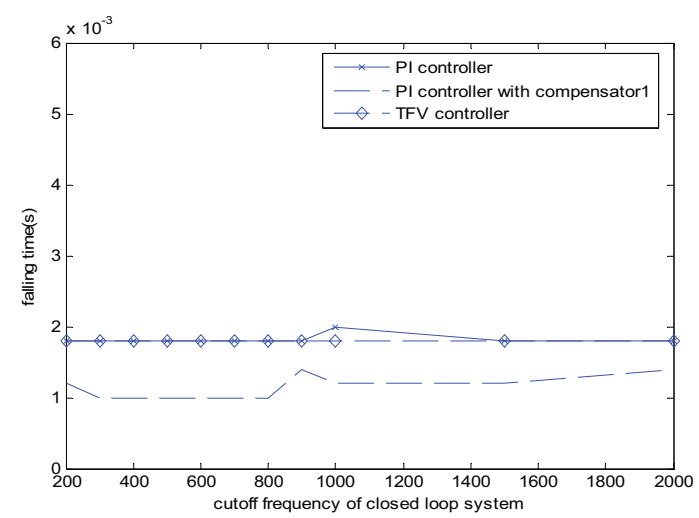

Fig. 13. Response Times Achieved with Different Control Laws

(ii) PI controller (in feedback COF form).

(iii) PI controller with compensator (feedback and feedforward controller in COF form).

We see from figure 13 that controller (iii) gives approximately $50 \%$ reduction in closed loop transient time independent of the closed loop bandwidth.

\section{A Brief introduction to Model Predictive CONTROL}

\section{A. Overview}

Model predictive control $(6 ; 7 ; 8 ; 9)$ has emerged as a very successful method to solve a wide range of problems in the process industries where the plant being controlled is "slow", (5). The method is being increasingly employed to control "fast" plants. It owes its success to its ability to (relatively easily) handle nonlinearity of the plant and to allow one to impose hard constraints on states and controls. These are requirements that are difficult to satisfy with other control methods. The penalty is that an open-loop optimal control problem (possibly nonlinear and constrained) has to be solved at each instant to obtain the control.

\section{B. Basic Ingredients of Deterministic MPC}

To allow for consideration of nonlinear systems, suppose the plant to be controlled is described by

$$
x^{+}=f(x, u)
$$

where $x$ and $u$ denote, respectively, the state and control and $x^{+}$the successor state, i.e. the state at the next sampling instant. Suppose the length of the horizon of the optimal control problem to be solved is $N$ and that the plant is subject to the control (input) constraint $u \in \mathbb{U}$ and state constraint $x \in \mathbb{X}$. We discuss the deterministic case. By "deterministic" we imply that there is no uncertainty in the system. In particular, the state $x$ is assumed to be known and there are no unknown disturbances. The optimal control problem (solved on line) requires that, for the known current state $x$ at time $t$, a constrained minimization be carried out with respect to the control sequence $\mathbf{u}=\{u(0), u(1), \ldots, u(N-1)\}$. Because the system is time-invariant, it is convenient to regard the initial time in the optimization problem to be 0 . The state at each time $k>0$ is a function of $(x, \mathbf{u})$. Hence, the cost $V_{N}(\cdot)$ is defined by

$$
V_{N}(x, \mathbf{u}):=\sum_{i=0}^{N-1} \ell(x(i), u(i))+V_{f}(x(N))
$$

in which $x(i)=\phi(i ; x, \mathbf{u})$ is the solution at time $i$ of the difference equation $x^{+}=f(x, u)$ when the initial state is $x$ and the control (input) sequence is chosen as $\mathbf{u}$. The minimization is carried out subject to the control and state constraints $u(i) \in \mathbb{U}$ and $x(i) \in \mathbb{X}$ for all $i \in\{0,1,2, \ldots, N-1\}$. It is also usual to impose a terminal state constraint of the form $x(N) \in X_{f}$. In this formulation, $V_{f}(\cdot)$ is the additional terminal cost (a control Lyapunov function in the neighbourhood of the set point) and $X_{f} \subseteq \mathbb{X}$ is the associated control positive invariant set. These are added to the optimal control problem as a mechanism to ensure closed-loop stability. The minimization yields the optimal control sequence

$$
\mathbf{u}^{0}(x)=\left\{u^{0}(0 ; x), u^{0}(1 ; x), \ldots, u_{N-1}(0, x)\right\}
$$

This is a function of the current state $x$ as well as the value function $V_{N}^{0}(x)=V_{N}\left(x, \mathbf{u}^{0}(x)\right)$. Model predictive control implements the solution in a "rolling horizon" fashion, i.e., we use only $u^{0}(0 ; x)$, the first element of this sequence, as the control $u$ to be applied to the plant. The control $u=\kappa_{N}(x):=u^{0}(0 ; x)$ applied to the plant is a function of the state. Hence a particular form of (nonlinear) state feedback control is obtained. When time advances one step, we measure (or estimate) the current state and repeat the calculation.

\section{Stability}

Subject to conditions on $f(\cdot), \ell(\cdot), V_{f}(\cdot)$ and $X_{f}$, then closed-loop stability (i.e. asymptotic or exponential) can be established (10) for the closed-loop system $x^{+}=f\left(x, \kappa_{N}(x)\right)$. This result is obtained using the value function $V_{N}^{0}(\cdot)$ as a Lyapunov function. Satisfaction of the conditions mentioned above ensures $V_{N}^{0}(\cdot)$ is zero at the target state, positive elsewhere and satisfies

$$
V_{N}^{0}\left(x^{+}\right) \leq V_{N}^{0}(x)-\ell\left(x, \kappa_{N}(x)\right), \quad x^{+}=f\left(x, \kappa_{N}(x)\right)
$$

Thus $V_{N}^{0}(\cdot)$ decreases along closed-loop trajectories and this ensures closed-loop stability. The analysis also shows that recursive feasibility is maintained, i.e., if the optimal control problem can be solved at the initial time, it can be solved at all future times. If the terminal cost is chosen appropriately (this is easy when the system is linear) and the set of states to be controlled restricted to a region around the target state, performance is identical to that obtained using an infinite horizon optimal control problem.

\section{Nonconvex problems}

If, the optimal control problem is non-convex (which is often the case if $f(\cdot)$ is nonlinear), then finding the global minimum of $V_{N}(\cdot)$ cannot be ensured. However, it has been 
shown (9) that determining, at each time, a feasible, rather than an optimal, solution yields, under mild conditions, a stabilising controller. Moreover, recursive determination of a feasible solution is always possible if the initial problem is feasible.

\section{E. Specialization to Linear Systems with Quadratic Cost}

1) Formulation: Here we study the special case where the system $f(\cdot)$ is linear as in (19) and (20) and the cost is quadratic.

Say that the current state estimate is $\hat{x}(0)$, then the future states over horizon $N$ can be predicted via

$$
\hat{\hat{x}}(i)=A^{i} \hat{x}(0)+\sum_{k=1}^{i} A^{i-k} B u(k-i+1)
$$

where $i=1, \ldots, N$. (Note that here we do not include a terminal cost.)

Let us assume a simple cost function of the form

$$
V_{N}(\hat{x}(0), \mathbf{u})=\sum_{k=1}^{N} \hat{\hat{e}}(k)^{2}+\lambda u(k-1)^{2}
$$

where $\hat{\hat{e}}(k)=C \hat{\hat{x}}(k)$. Clearly this is a quadratic function of $\hat{x}(0)$ and $\mathbf{u}=\{u(0), u(1), \ldots, u(v)\}$.

Now the cost function (54) can be expressed in matrix notation as

$$
V_{N}(\hat{x}(0), \mathbf{u})=(\mathcal{H} \mathbf{u}+\mathbf{r})^{T}(\mathcal{H} \mathbf{u}+\mathbf{r})+\lambda \mathbf{u}^{T} \mathbf{u}
$$

where

$$
\begin{gathered}
\mathcal{H}=\left[\begin{array}{ccc}
C B & & 0 \\
& \ddots & \\
C A^{N-1} B & \cdots & C B
\end{array}\right] \\
\mathbf{u}=\left[\begin{array}{c}
u(0) \\
\vdots \\
u(N-1)
\end{array}\right] ; \mathbf{r}=\left[\begin{array}{c}
C A \\
\vdots \\
C A^{N}
\end{array}\right] \hat{x}(0)
\end{gathered}
$$

2) Unconstrained Solution: In the absence of constraints, we can easily find $\mathbf{u}$ that minimizes (55) namely

$$
\mathbf{u}=-\left[\mathcal{H}^{T} \mathcal{H}+\lambda I\right]^{-1} \mathcal{H}^{T} \mathbf{r}
$$

3) Constrained Solution: In the presence of constraints, we need to minimize (55) subject to satisfaction of the constraints.

When rolling horizon optimization is used, we implement $u(0)$ only. We then measure (or estimate) a new state $\hat{x}(1)$, reset the current time to zero and repeat the problem. At each step, we apply

$$
u(0)=\left[\begin{array}{llll}
1 & 0 & \ldots & 0
\end{array}\right] \mathbf{u}^{*}
$$

where $\mathbf{u}^{*}$ is the vector optimizing the cost function.
4) Horizon 1: In many applications (including power electronics and drives) the sampling rate is often very high (e.g. $\triangle_{s}$ is typically chosen as a fraction of a millisecond). In this case, it is usually prohibitive to use large values for the horizon $N$. Hence it is common to use small horizons e.g. unity.

When there are no constraints, then it can be seen, by specializing (58) to horizon 1 , that the input is given by

$$
u(0)=-K \hat{x}(0)
$$

where, in this special case,

$K=\left[B^{T} C^{T} C B+\lambda I\right]^{-1} C B C A$.

When constraints are added, it is relatively simple to modify the solution as shown below. Let $u^{u c}(0)$ be the solution to the unconstrained problem. Then the cost can be factored as

$$
\begin{aligned}
& V_{N}(\hat{x}(0), \mathbf{u}) \\
& =\left[u(0)-u^{u c}(0)\right]^{T}\left[B^{T} C^{T} C B+\lambda I\right]\left[u(0)-u^{u c}(0)\right] \\
& \quad+\text { constant }
\end{aligned}
$$

Next say the we require that $u(0) \in \mathbb{U}$, a given constraint set. (Note that this set can be convex or finite.)

We factor the Hessian in the above expression as follows

$$
D^{T} D \doteq\left[B^{T} C^{T} C B+\lambda I\right]
$$

and define

$$
\begin{aligned}
\bar{u} & =D u(0) \\
\bar{u}^{u c} & =D u^{u c}(0)
\end{aligned}
$$

We also transform the allowable constraint set by defining

$$
\overline{\mathbb{U}}=D \mathbb{U}
$$

Then we see that

$$
V_{N}(\hat{x}(0), \mathbf{u})=\left(\bar{u}-\bar{u}^{u c}\right)^{T}\left(\bar{u}-\bar{u}^{u c}\right)+\text { constant }
$$

We also see that the constrained optimization problem is solved by finding the closest value (in a Euclidean sense) within $\overline{\mathbb{U}}$ to the unconstrained solution $\bar{u}^{u c}$.

In summary, we have that the constrained optimal solution is

$$
u(0)=D^{-1}\left[\bar{Q}\left\{D u^{u c}(0)\right\}\right]=Q\left[u^{u c}(0)\right]
$$

where $\bar{Q}$ and $Q$ denote a simple "nearest neighbour" quantizer and a generalized quantizer respectively.

We thus see that the horizon 1 solution is defined via the generalized "projection" quantizer given in (65). Moreover, it is then immediately apparent that the horizon 1 solution is a special case of the COF principle described in section III-G where the "quantizer" takes the form given in (65). For this reason, using the term MPC in this context is perhaps misleading. We thus prefer the term "COF" rather than "MPC" for the horizon 1 case, see (15), (16), (21). Extensions to longer horizons are discussed in (17), (22). 


\section{F. The Spectra of Uncertainty}

We return to the general formulation described in sections $\mathrm{V}-\mathrm{B}$ and V-C. Unfortunately, the core stability result assumes no uncertainty (i.e., no disturbances or modelling error).

If uncertainty is present, the equivalence between openloop and feedback control disappears and the proven stability properties of deterministic model predictive control are lost. Thus it is no longer true that the solution to an open-loop optimal control problem (modified to compensate for its finite horizon) provides optimal control of the uncertain system. We could envisage a "feedback" solution to account for uncertainty; however, an optimal control problem that provides the optimal "feedback" solution would be impossibly complex. Hence, as in adaptive control, 'smart' non-optimal solutions have to be devised. What is often done in practice is to obtain model predictive control for the nominal system, i.e to ignore uncertainty in computing the on-line control action. This can give satisfactory control (but is not guaranteed to do so). To see what might happen, consider the simple case when the state is measured but a disturbance, $w$, enters additively; the system is then described by

$$
x^{+}=f(x, u)+w
$$

Since now $x^{+}=f\left(x, \kappa_{N}(x)\right)+w$ rather than $x^{+}=$ $f\left(x, \kappa_{N}(x)\right)$, the evolution equation for the value function $V_{N}^{0}(\cdot)$ (of the nominal control problem) satisfies

$$
V_{N}^{0}\left(x^{+}\right)=V_{N}^{0}(x)-\ell\left(x, \kappa_{N}(x)\right)+\delta(x, w)
$$

The extra term, $\delta(x, w):=V_{N}^{0}\left(f\left(x, \kappa_{N}(x)\right)+w\right)-$ $V_{N}^{0}\left(f\left(x, \kappa_{N}(x)\right)\right.$, may well exceed $\ell\left(x, \kappa_{N}(x)\right)$ in magnitude so that a decrease of the candidate Lyapunov function is no longer ensured. Also, recursive feasibility is no longer guaranteed.

To overcome these features of nominal MPC various schemes have been proposed. One proposal, called tube model predictive control (9), uses nominal MPC to determine a nominal trajectory from the current set point to the next set point and local feedback to confine the trajectories of the uncertain system to remain in a specified neighbourhood of the nominal trajectory. If the system $f(\cdot)$ is linear and if $z(i)$ and $v(i)$ are the state and control of the nominal system at time $i$, the control $u(i)$ applied to the plant is $u(i)=v(i)+K(x(i)-z(i))$ where $x(i)$ is the current state of the plant. If the disturbance, $w$, is bounded, a bounded set $S$ may be computed such that the state $x(i)$ of the plant satisfies $x(i) \in z(i)+S$ for all $i$ and all possible disturbance sequences. The state $z(i)$ of the nominal system converges to the set point $x^{*}$ and the state $x(i)$ of the uncertain system converges to the set $x^{*}+S$; once the state $x(i)$ enters the set $x^{*}+S$, it remains there. With this knowledge, nominal MPC may be designed to ensure that the uncertain system satisfies all constraints. If the state is not directly measured, as is commonly the case, a similar procedure is used to control the state estimator. The latter can be viewed as another "uncertain system" with measured state.

\section{G. Quantised Control}

The stability results described above assume the control $u$ lies in, say, $\mathbb{U}$ where the input constraint set $\mathbb{U}$ is a convex subset of, say, $\mathbb{R}^{m}$. In power electronics and other applications, $\mathbb{U}$ is typically a finite subset of $\mathbb{R}^{m}$, e.g. $\mathbb{U}=\left\{u_{1}, u_{2}, \ldots, u_{m}\right\}$ is the set of voltages, say, that may be generated by the power electronics. A finite alphabet $\mathbb{U}$ raises new problems regarding a proof of stability. Some of these problems are discussed below.

\section{Vi. Certificates of Stability}

\section{A. General Comments}

It is desirable, but not always possible, to imbue a control law with a "certificate of stability".

One way to achieve staility is by having a Control Lyapunov Function. In the context of Model Predictive Control, stability can be achieved by adding to the optimal control problem a terminal cost function that is a global Control Lyapunov Function or by adding a terminal cost function that is merely a local Control Lyapunov Function provided that a terminal constraint is also added such that the local Control Lyapunov Function is valid within the terminal constraint set. This, in turn, raises the issue of "feasibility", i.e. the ability to steer the system from its initial state into the terminal constraint set. This is where a longer horizon can be useful since it offers greater flexibility (i.e. more control moves) to reach the terminal set.

Several special cases facilitate the search for a Control Lyapunov Function. Two such cases are discussed below.

(i) When the $B$ matrix in the system description is invertible. The key point about this case is that it is always possible to steer any initial state lying sufficiently close to the target state $x^{*}$ to a point "closer" to the target state (even in one step). Hence, in this case, there exists a wide range of local Control Lyapunov Functions such as the function $\left|x-x^{*}\right|$ used in some power electronic applications. In this case, the Control Lyapunov Functions is local because the control constraint restricts the range of states that can be moved "closer" to the target state.

(ii) When the system is open loop stable. The key point about this case is that one can choose the Control Lyapunov Function (CLF) to be the function $V_{f}(x):=(1 / 2)(x-$ $\left.x^{*}\right)^{\prime} P\left(x-x^{*}\right)$ where $x^{*}$ is the target state and $P$ is the solution of the Lyapunov equation

$$
P=A^{T} P A+\Omega
$$

where $\Omega$ is any positive definite symmetric matrix. With this choice for $V_{f}(\cdot)$, the control $u=u^{*}$, which steers the system to $x^{*}$, causes a reduction in the CLF and any control that minimizes $V_{f}\left(x^{+}\right), x^{+}=A x+B u$, will decrease the CLF more and steer the system to $x^{*}$ even more quickly. This CLF is global and this makes it preferable to the local CLF discussed in (i). Invertibility of $B$ is also not required in this case. 
Obtaining a control by minimizing $V_{f}\left(x^{+}\right)$with respect to $u$, is actually equivalent to an old form of control in which a Control Lyapunov Function (CLF)is directly employed to obtain a stabilising controller. However, MPC provides extra freedom. For example, a stabilising controller may also be obtained by employing the cost $V_{1}(x, u):=\ell(x, u)+V_{f}(A x+$ $B u)$ in place of $V_{1}(x, u):=V_{f}(A x+B u)$. As before, $V_{f}(\cdot)$ should be a CLF. The function $\ell(\cdot)$, which should satisfy some simple conditions, may be used to improve performance if $V_{f}(\cdot)$ is chosen appropriately. Larger horizons would provide even more flexibility.

\section{B. Quantization}

Quantization introduces an extra layer of difficulty over those described above. For example, with finite alphabet control it is not a-priori obvious that one has the flexibility to the steer the state to a desired target $x^{*}$ that is an equilibrium state in the sense that there exists a control $u^{*}$ satisfying $x^{*}=A x^{*}+B u^{*}$ even if $B$ is invertible. Difficulty arises if the control $u *$ does not lie in the finite alphabet. This problem requires a more detailed analysis.

One option would be to relax the problem by ignoring the discrete nature of $\mathbb{U}$, i.e. $\mathbb{U}$ is replaced in the optimal control problem by $\mathbb{V}$ which is defined to be the convex hull of $\mathbb{U}$ yielding a control $u$. The control actually applied to the plant is then $u_{\text {true }}=Q(u)$ where $Q$ is a generalized quantizer. The quantizer noise $q:=u_{\text {true }}-u$ is bounded so that the plant, if it is linear, is now described by

$$
x^{+}=A x+B u_{\text {true }}+B q
$$

and is an uncertain system with bounded additive disturbance $w=B q$. This can be treated by using techniques from the emerging theory of robust model predictive control - see (9), (11), (12), (13).

\section{Fusion Control}

It has been suggested above that one way to treat quantization is to transform the optimization problem into one with unquantized control and additive bounded noise. Of course, noise comes in different "flavours". Hence, there may be value in combining some of the ideas used in section III with Model Predictive Control. We term such strategies "Fusion Control" since the goal is to fuse modern and traditional ideas.

\section{The SynRM Revisited}

The voltage and torque equations for a three-phase synchronous reluctance motor in the rotating DQ reference frame are given in section II. If the state $x$ for the current loop is defined to be the vector $\left(i_{d}, i_{q}\right)$, then the system satisfies:

$$
\begin{aligned}
\dot{x} & =\bar{A}\left(\omega_{0}\right) x+\bar{B} u \\
y & =c(x)
\end{aligned}
$$

where

$$
\bar{A}:=\left[\begin{array}{cc}
-\alpha_{1} & \beta_{1}\left(\omega_{0}\right) \\
-\beta_{2}\left(\omega_{0}\right) & -\alpha_{2}
\end{array}\right], \quad \bar{B}:=\left[\begin{array}{cc}
1 / L_{1} & 0 \\
0 & 1 / L_{2}
\end{array}\right]
$$

and

$$
c(x):=(3 p / 2)\left(L_{d}-L_{q}\right) x_{1} x_{2}
$$

where $\alpha_{1}:=R_{s} / L_{d}, \alpha_{2}:=R_{s} / L_{q}, \beta_{1}\left(\omega_{0}\right):=\left(L_{d} / L_{q}\right) \omega_{0}$, $\beta_{2}\left(\omega_{0}\right):=\left(L_{q} / L_{d}\right) \omega_{0}, u$ denotes the voltage vector $\left(v_{d}, v_{q}\right)$, $\omega_{0}$ the 'electrical' angular velocity, $y$ is the output torque and $p$ is the number of pole pairs in the motor. The open loop characteristic equation is

$$
\phi(s)=s^{2}+\left(\alpha_{1}+\alpha_{2}\right) s+\alpha_{1} \alpha_{2}+\omega_{0}^{2}
$$

This characteristic polynomial is lightly damped if $\omega_{0}$ is large. The outer loop of the speed control system provides a reference current $x^{*}$ for an inner loop; $x_{1}^{*}$ is constant at a value that provides maximum flux while $x_{2}^{*}$ is varied by the outer loop to achieve the desired angular velocity $\omega_{0}^{*}$. The discretetime model for the inner loop corresponding to a sampling period of $\triangle_{s}$ seconds is:

$$
x^{+}=A x+B u
$$

where $A:=\exp \left(\bar{A} \triangle_{s}\right)$ and $B:=\int_{0}^{T_{s}} \exp (\bar{A} t) \bar{B} d t$. This translates the problem into a form where one could use MPC. However, the core problem is the finite alphabet nature of the control. Hence MPC with horizon length $N$ requires choosing, via optimization, a control sequence lying in $\mathbb{U}^{N}$. This is a difficult problem if $N$ is large. If we use a horizon length $N=1$, then the problem becomes much simpler as discussed in section V-E4. Moreover, for this particular problem, $\bar{B}$ and hence $B$, are nonsingular and $A$ is stable. Hence, the ideas discussed in section VI-A (i) and (ii) are potentially useful.

\section{Challenges And Opportunities}

\section{A. Horizon Length}

Much of the literature on MPC for power electronic applications uses, for computational reasons, a horizon length $N$ of unity although there are a few exceptions (4), (36). As we have shown in section VI-B, it is possible to establish closed loop stability with $N=1$ e.g., if the system being controlled is invertible in one step or is open loop stable which is the case in some applications. There is a need to investigate whether significantly better performance can be obtained with longer horizons together with suitable choice of the stage cost $\ell(\cdot)$, state constraint, and terminal cost. If a (global) control Lyapunov is available, as is often the case, a terminal constraint is not required. Indeed much of the power of MPC arises from its flexibility in choice of cost function and from its ability to handle hard constraints. Longer horizons may help with performance objectives such as reduction of switching frequency (36). It would also be helpful to know under what conditions a horizon length of unity yields good performance.

\section{B. Quantization Effects}

There appear to be two distinct possibilities in MPC for handling the quantization that is induced by the switching converters. One, that seems to be preferred in the current literature, is to pose the optimal control problems as minimizing a 
cost subject to the constraint $\mathbf{u} \in \mathbb{U}^{N}$ where $\mathbb{U}$ is the discrete set specifying permissible switched voltages. This approach causes theoretical difficulties discussed above and requires solution of an integer program. The second alternative, also discussed above, is to perform the minimization over $\mathbb{V}^{N}$ where $\mathbb{V}$ is the convex hull of $\mathbb{U}$. The resultant optimal $u$ is then quantised and the nearest neighbour to $u$ applied to the plant; alternatively PWM or SVF modulation may be employed as is done when PI control is used. The relaxed problem is simpler, being a conventional quadratic or nonlinear programme. This permits a traditional analysis of closed loop stability. It would be interesting to compare the performances of these two approaches. Also, the potential advantages of using feedback around the quantizer to modify the frequency spectrum of the quantization errors should be explored.

\section{Robustness}

Existing design techniques for unconstrained control problems permit a wide range of robustness issues such as undermodelling, unknown parameters, unknown states, the effect of disturbances to be addressed. Unfortunately, the same is not true for MPC since, as pointed out above, the solution to the open loop optimal control problem solved online is not equivalent to the feedback solution. A consequence is that nominal MPC, which ignores uncertainty and is widely used, may have poor robustness properties. Current research is searching for modifications to nominal MPC that ensure robustness. Some proposals have been made for the case when the uncertainty takes the form of an unknown, but bounded, disturbance and also when the state has to be estimated. Recent work on unmodelled dynamics is given in (11) to (14). However the issues of undermodelling and unknown parameters have yet to be fully resolved.

\section{Control Architecture}

In classical control several different architectures have been employed to good effect. One of these is the multi-degree of freedom controller architecture as described in section III-F. It would be interesting to know if MPC would benefit from more complex architectures than the single loop that is currently employed. A related question is the use of 'inner' and 'outer' loops. In some applications, MPC is used for an inner current or torque loop and a conventional controller for an outer speed loop. This raises the question as to whether or not it would be advantageous to dispense with the inner loop and use a single model predictive controller for the outer loop? Another alternative would be to employ model predictive controllers in both the inner and outer loop.

\section{E. Cost Function}

The cost function for Power Electronic Applications typically has a "standard" form which assigns penalty coefficients to each component of the output error. This is somewhat arbitrary and subjective process. Development of a unified approach to cost function design would be beneficial. Variable or adaptive cost functions that achieve best performance under varying operating conditions and alternative design requirements would be useful.

\section{CONCLUSION}

This paper has reflected on the challenges and opportunities that exist when applying advanced control to power electronics and drives. We have reviewed various aspects of classical control and model predictive control. We have also commented on issues that arise when these techniques are applied to switched systems and raised a number of open problems and challenges.

\section{REFERENCES}

[1] M. P. Kazmierowski, R. Krishnan, and F. Blaabjerg, Control in Power Electronics. Academic Press, 2002.

[2] N. Mohan, T. M. Underland, and W. P. Robbins, Power Electronics. Hoboken, NJ: Wiley, 1995.

[3] D. G. Holmes and T. A. Lipo, Pulse Width Modulation for Power Converters. Piscataway, NJ: IEEE Press, 2003.

[4] P. Cortés, M. P. Kazmierowski, R. M. Kennel, D. E. Quevedo, and J. Rodríguez, "Predictive control in power electronics and drives," IEEE Trans. Ind. Electron., vol. 55, pp. 4312-4324, Dec. 2008.

[5] S. J. Qin and T. A. Badgwell, "A survey of industrial model predictive control technology," Contr. Eng. Pract., vol. 11, pp. 733764, 2003.

[6] J. M. Maciejowski, Predictive Control with Constraints. Englewood Cliffs, N. J.: Prentice-Hall, 2002.

[7] E. F. Camacho and C. Bordons, Model Predictive Control. New York, N.Y.: Springer-Verlag, 1999.

[8] G. C. Goodwin, M. M. Serón, and J. A. De Doná, Constrained Control \& Estimation - An Optimization Perspective. London: Springer Verlag, 2005.

[9] J. B. Rawlings and D. Q. Mayne, Model Predictive Control: Theory and Design. Madison, WI: Nob Hill Publishing, 2009.

[10] D. Q. Mayne, J. B. Rawlings, C. V. Rao, and P. O. M. Scokaert, "Constrained model predictive control: Optimality and stability," Automatica, vol. 36, pp. 789-814, June 2000.

[11] C. Løvaas, M. M. Serón, and G. C. Goodwin, "Robust output feedback model predictive control for systems with unstructured uncertainty," Automatica, vol. 44, pp. 1933-1943, 2008.

[12] C. Løvaas, M. M. Serón, and G. C. Goodwin, "Inverse minimax optimality of model predictive control policies," Syst. \& Contr. Lett., July 2009. accepted for publication.

[13] C. Løvaas, M. M. Serón, and G. C. Goodwin, "Robust output feedback MPC with integral action," IEEE Trans. Automat. Contr., Oct. 2009. accepted for publication.

[14] D. E. Quevedo and D. Nešić, "Input-to-state stability of packetized predictive control over unreliable networks affected by packet-dropouts," IEEE Trans. Automat. Contr., 2010. accepted for publication.

[15] G. C. Goodwin, D. E. Quevedo, and D. McGrath, "Movinghorizon optimal quantizer for audio signals," J. Audio Eng. Soc., vol. 51, pp. 138-149, Mar. 2003.

[16] D. E. Quevedo and G. C. Goodwin, "Multistep optimal analogto-digital conversion," IEEE Trans. Circuits Syst. I, vol. 52, pp. 503-515, Mar. 2005.

[17] D. E. Quevedo, G. C. Goodwin, and J. A. De Doná, "Finite constraint set receding horizon quadratic control," Int. J. Robust Nonlin. Contr., vol. 14, pp. 355-377, Mar. 2004.

[18] A. Gersho and R. M. Gray, Vector Quantization and Signal Compression. Boston, MA: Kluwer Academic, 1992.

[19] G. C. Goodwin, D. E. Quevedo, and E. I. Silva, "Architectures and coder design for networked control systems," Automatica, vol. 44, pp. 248-257, Jan. 2008. 
[20] E. I. Silva, G. C. Goodwin, and D. E. Quevedo, "Control system design subject to SNR constraints," Automatica, vol. 46, pp. 428 436, Feb. 2010.

[21] J. A. De Doná, G. C. Goodwin, and M. M. Serón, “Anti-windup and model predictive control: Reflections and connections," European J. Contr., vol. 6, no. 5, pp. 467-477, 2000.

[22] D. E. Quevedo, C. Müller, and G. C. Goodwin, "Conditions for optimality of naïve quantized finite horizon control," Int. J. Contr., vol. 80, pp. 706-720, May 2007.

[23] S. Kouro, P. Cortés, R. Vargas, U. Ammann, and J. Rodríguez, "Model predictive control-A simple and powerful method to control power converters," IEEE Trans. Ind. Electron., vol. 56, pp. 1826-1838, June 2009.

[24] A. Linder and R. Kennel, "Model predictive control for electrical drives," in Proc. IEEE Power Electronics Specialists Conference (PESC), (Recife, Brazil), pp. 1793-1799, 2005.

[25] J. R. Rodríguez, J. Pontt, C. Silva, P. Correa, P. Lezana, and P. Cortés, "Predictive current control of a voltage source inverter," IEEE Trans. Ind. Electron., vol. 54, pp. 495-503, Feb. 2007.

[26] G. Perantzakis, F. Xepapas, S. Papathanassiou, and S. N. Manias, "A predictive current control technique for three-level NPC voltage source inverters," in Proc. IEEE Power Electronics Specialists Conference (PESC), pp. 1241-1246, 2005.

[27] R. Vargas, P. Cortés, U. Ammann, J. Rodríguez, and J. Pontt, "Predictive control of a three-phase neutral point-clamped inverter," IEEE Trans. Ind. Electron., vol. 54, pp. 2697-2705, Oct. 2007.

[28] G. Perantzakis, F. Xepapas, and S. N. Manias, "Efficient predictive control technique for multilevel voltage source inverters," in Proc. Eur. Conf. Power Electron. Appl., Sept. 2005.

[29] S. Muller, U. Ammann, and S. Rees, "New time-discrete modulation scheme for matrix converters," IEEE Trans. Ind. Electron., vol. 52, pp. 1607-1615, Dec. 2005.

[30] G. Papafotiou, T. Geyer, and M. Morari, "A hybrid model predictive control approach to the direct control problem of induction motors," Int. J. Robust Nonlin. Contr., vol. 17, no. 17, pp. 1572-1589, 2007.

[31] P. Lezana, R. P. Aguilera, and D. E. Quevedo, "Model predictive control of an asymmetric flying capacitor converter," IEEE Trans. Ind. Electron., vol. 56, pp. 1839-1846, June 2009.

[32] J. Huang and K. A. Corzine, "Extended operation of flying capacitor multilevel inverters," IEEE Trans. Power Electron., vol. 21, pp. 140-147, Jan. 2006.

[33] P. Cortés, J. Rodríguez, D. E. Quevedo, and C. Silva, "Predictive current control strategy with imposed load current spectrum," IEEE Trans. Power Electron., vol. 23, pp. 612-618, Mar. 2008.

[34] P. Cortés, G. Ortiz, J. I. Yuz, J. Rodríguez, S. Vázquez, and L. G. Franquelo, "Model predictive control of an inverter with output LC-filter for UPS applications," IEEE Trans. Ind. Electron., vol. 56, pp. 1875-1883, June 2009.

[35] H. Miranda, P. Cortés, J. I. Yuz, and J. Rodríguez, "Predictive torque control of induction machines based on state-space models," IEEE Trans. Ind. Electron., vol. 56, pp. 1916-1924, June 2009.

[36] T. Geyer, G. Papafotiou, and M. Morari, "Model predictive direct torque control-part I: Concept, algorithm and analysis," IEEE Trans. Ind. Electron., vol. 56, pp. 1894-1905, June 2009.

[37] T. Geyer, "Generalized model predictive direct torque control: Long prediction horizons and minimization of switching losses," in Proc. IEEE Conf. Decis. Contr., (Shanghai, China), Dec. 2009.

[38] G. Papafotiou, J. Kley, K. G. Papadopoulos, P. Bohren, and M. Morari, "Model predictive direct torque control-Part II: Implementation and experimental evaluation," IEEE Trans. Ind. Electron., vol. 56, pp. 1906-1915, June 2009.

[39] D. Nešić and L. Grüne, "A receding horizon control approach to sampled-data implementation of continuous-time controllers," Syst. \& Contr. Lett., vol. 55, pp. 660-672, 2006.
[40] D. E. Quevedo, R. P. Aguilera, M. A. Pérez, and P. Cortés, "Finite control set MPC of an AFE rectifier with dynamic references," in Proc. IEEE Conf. on Industrial Technology, 2010.

[41] A. Bemporad, "Reference governor for constrained nonlinear systems," IEEE Trans. Automat. Contr., vol. 43, no. 3, pp. 415419, 1998.

[42] R. P. Aguilera, D. E. Quevedo, T. J. Summers, and P. Lezana, "Predictive control algorithm robustness for achieving fault tolerance in multicell converters," in Proc. 34th Annual Conf. IEEE Ind. Electron. Soc. (IECON'08), pp. 3302-3308, 2008.

[43] T. Matsuo and T. A. Lipo. Rotor design optimization of a synchronous reluctance machine. IEEE Transactions on Energy Conversion, 9(2):359-365, 1994.

[44] C. E. Coates, D. Platt, and V. J. Gosbel. Performance evaluation of a nine-phase synchronous reluctance drive. In Proceedings of IEEE-IAS Annual Meeting, volume 3, pages 2041-2047, Chicago, October 2001.

[45] G. C. Goodwin, S. E. Graebe, and M. Salgado. Principles of control system design. Prentice Hall, Englewood Cliffs, NJ, 1997.

[46] L. Xu, X. Xu, T. A. Lipo, and D. W. Novotny:1991. Vector control of a synchronous reluctance motor including saturation and iron loss. IEEE Transactions on Industry Applications, 27(5):977-985, 1991.

[47] T. A. Lipo. Synchronous reluctance machines - a viable alternative for AC drives? Electric machines and Power Systems, 19:659-671, 1991.

[48] P. Vas. Sensorless Vector and Direct Torque Control. Oxford university Press, 2003. 